\title{
The Chromatin of Candida albicans Pericentromeres Bears Features of Both Euchromatin and Heterochromatin
}

\author{
Verónica Freire-Benéitez, R. Jordan Price and Alessia Buscaino* \\ School of Biosciences Canterbury Kent, University of Kent, Canterbury, UK
}

Centromeres, sites of kinetochore assembly, are important for chromosome stability and integrity. Most eukaryotes have regional centromeres epigenetically specified by the presence of the histone H3 variant CENP-A. CENP-A chromatin is often surrounded by pericentromeric regions packaged into transcriptionally silent heterochromatin. Candida albicans, the most common human fungal pathogen, possesses small regional centromeres assembled into CENP-A chromatin. The chromatin state of C. albicans pericentromeric regions is unknown. Here, for the first time, we address this question.

OPEN ACCESS

Edited by: Ana Traven

Monash University, Australia

Reviewed by:

Laura Rusche,

State University of New York

at Buffalo, USA

Kristin Scott,

Duke University, USA

${ }^{*}$ Correspondence:

Alessia Buscaino

a.buscaino@kent.ac.uk

Specialty section:

This article was submitted to Fungi and Their Interactions,

a section of the journal

Frontiers in Microbiology

Received: 29 February 2016 Accepted: 05 May 2016

Published: 19 May 2016

Citation:

Freire-Benéitez V, Price Ru and Buscaino A (2016) The Chromatin of Candida albicans Pericentromeres Bears Features of Both Euchromatin

and Heterochromatin.

Front. Microbiol. 7:759.

doi: 10.3389/fmicb.2016.00759
We find that $C$. albicans pericentromeres are assembled into an intermediate chromatin state bearing features of both euchromatin and heterochromatin. Pericentromeric chromatin is associated with nucleosomes that are highly acetylated, as found in euchromatic regions of the genome; and hypomethylated on $\mathrm{H} 3 \mathrm{~K} 4$, as found in heterochromatin. This intermediate chromatin state is inhibitory to transcription and partially represses expression of proximal genes and inserted marker genes. Our analysis identifies a new chromatin state associated with pericentromeric regions.

Keywords: epigenetics, chromatin, centromere, Candida albicans, heterochromatin

\section{INTRODUCTION}

The centromere is the cis-acting DNA site of kinetochore assembly and spindle attachment during chromosome segregation in mitosis and meiosis. Centromeric regions have a different organization across different species. Some organisms, such as the budding yeast Saccharomyces cerevisiae, have "point" centromeres while other organisms, such as the fission yeast, the fruit fly and human, have "regional" centromeres (Buscaino et al., 2010). Point centromeres are only $\sim 125$ bp long and include specific DNA binding sites necessary for centromere function (Westermann et al., 2007). Regional centromeres span large DNA domains ( $\sim 10$ to $10,000 \mathrm{~kb})$ and do not contain a specific DNA sequence but are epigenetically specified by the presence of the histone $\mathrm{H} 3$ variant, CENP-A (also termed Cse4 and CENH3) (Buscaino et al., 2010). Regional centromeres are often associated with repetitive elements. The structure and organization of centromereassociated DNA repeats varies across organisms. For example, human centromeres are composed of tandem arrays of 171 alpha-satellite repeats and, in Drosophila melanogaster, centromeric DNA contains short repetitive elements interspersed with transposable elements (Buscaino et al., 2010). In the yeast Schizosaccharomyces pombe and the fungal pathogen Candida tropicalis, centromeres are organized in a CENP-A-containing central/mid core domain flanked by inverted repeats 
(IRs) whose sequences are conserved across centromeres (Buscaino et al., 2010; Chatterjee et al., 2016). In both organisms, these IRs are important for de novo CENP-A deposition on a plasmid containing the central core sequence (Baum et al., 1994; Chatterjee et al., 2016). Pericentromeric regions are usually assembled into transcriptionally silent heterochromatin that is required for establishment of CENP-A chromatin and for faithful chromosome segregation (Bernard et al., 2001; Nonaka et al., 2002; Folco et al., 2008). At these locations, heterochromatin is hypoacetylated at Lysine 9 of Histone $\mathrm{H} 3$ (H3K9) and Lysine 16 of Histone $\mathrm{H} 4$ (H4K16). Heterochromatic regions are also hypomethylated at Lysine 4 of Histone $\mathrm{H} 4$ (H3K4) and methylated at H3K9 (Strahl and Allis, 2000; Kouzarides, 2007). Histone modifiers control this modification state: for example the histone deacetylase (HDAC) Sir2 deacetylates H3K9 and/or H4K16, the histone methyltransferase Set1 methylates H3K4 and the histone methyltransferase $\mathrm{Su}(\mathrm{var}) 3-9$ methylates H3K9 (Shankaranarayana et al., 2003; Wirén et al., 2005; Bühler and Gasser, 2009; Kueng et al., 2013). Although pericentromeric heterochromatin is usually associated with pericentromeric regions, this repressive chromatin state is not absolutely required for centromere function and faithful chromosome segregation. For example, in Candida lusitaniae pericentromeric regions are not assembled into heterochromatin (Kapoor et al., 2015). Given the diversity of centromere structure across eukaryotes, it is important to analyze centromere organization in a variety of organisms.

Candida albicans, the most common human fungal pathogen, is an ideal system to investigate diversity and structure of centromeres because it possess regional centromeres that are much smaller and simpler than other regional centromeres (Sanyal et al., 2004; Baum et al., 2006; Mishra et al., 2007). Each of the 8 C. albicans diploid chromosomes has a relatively small regional centromere $(2-4 \mathrm{~kb})$ assembled into CENP-A chromatin (Baum et al., 2006). The organization and sequence of pericentromeric regions differs at each centromere (Figure 1A). Centromeres on chromosome 1, 4, 5, 6, and $\mathrm{R}$ are similar to centromeres of the fission yeast Schizosaccharomyces pombe where IRs flank the CENP-A containing domain. Contrary to $S$. pombe, the sequence of these repetitive elements is not conserved across centromeres. On chromosome 2 and 3 Long Terminal Repeats (LTRs) are found within $\sim 3 \mathrm{~kb}$ of the CENPA containing domain. Centromere on chromosome 7 does not have obvious repeats nearby (Mishra et al., 2007; Ketel et al., 2009). Therefore, 7 of the 8 pericentromeric regions are associated with DNA repeats. Several lines of evidence suggest that, in C. albicans, pericentromeric repeats are important for centromere function and/or establishing centromere identity. First of all, despite the lack of conservation in the DNA sequence, repetitive DNA is also associated with centromere of other Candida species such as C. dubliniensis (Padmanabhan et al., 2008). In addition, following deletions of endogenous centromeres, C. albicans neocentromeres form efficiently and are often assembled in proximity to DNA repeats (Ketel et al., 2009). However, the lack of repetitive elements surrounding the centromere on chromosome 7 argue against a role of DNA repeats in centromere function. In many eukaryotes, pericentromeric regions are assembled into transcriptionally silent heterochromatin. It is possible that, despite the lack of a conserved DNA sequence and/or DNA feature, the common feature of $C$. albicans pericentromeric regions is a specific chromatin structure resembling heterochromatin. Here, we address this question.

The C. albicans epigenome, similarly to $S$. cerevisiae, lacks $\mathrm{Su}$ (var) 3-9 orthologs, hence H3 K9 methylation is absent in both C. albicans and S. cerevisiae.

We have recently shown that, in C. albicans, transcriptionally silent heterochromatin is assembled at the ribosomal DNA ( $r D N A)$ locus and at telomeres (Freire-Benéitez et al., 2016). At these locations, heterochromatin is typified by nucleosomes that are hypoacetyled and hypomethylated on H3K4. The histone deacetylateses Sir2 (orf 19.1992) is required to maintain this repressive epigenetic state via hypoacetylation of $\mathrm{H} 3 \mathrm{~K} 9$ and H4K16 (Freire-Benéitez et al., 2016).

In this study, we investigate the chromatin state associated with $C$. albicans pericentromeric repeats. We find that pericentromeric regions are assembled into an intermediate chromatin state bearing features of both euchromatin (high histone acetylation) and heterochromatin (hypomethylation of $\mathrm{H} 3 \mathrm{~K} 4$ ). This intermediate chromatin state is associated with a weak transcriptionally silent environment that partially represses expression of proximal genes and inserted marker genes. Our analysis identifies a new chromatin state associated with pericentromeric regions.

\section{MATERIALS AND METHODS}

\section{Growth Conditions}

Yeast cells were cultured in rich medium (YPAD) containing extra adenine $(0.1 \mathrm{mg} / \mathrm{ml})$ and extra uridine $(0.08 \mathrm{mg} / \mathrm{ml})$, complete SC medium (Formedium ${ }^{\mathrm{TM}}$ ), or SC Drop-Out media (Formedium ${ }^{\mathrm{TM}}$ ). Cells were grown at 30 or $39^{\circ} \mathrm{C}$ as indicated.

\section{Yeast Strain Construction}

Strains are listed in Supplementary Table S1. Integration and deletion of genes were performed using plasmids containing marker genes for substitution or integration at endogenous locus as previously described (Wilson et al., 1999). Transformation was performed by electroporation (Gene Pulser ${ }^{\mathrm{TM}}$, Bio-Rad) using the protocol described in (De Backer et al., 1999). URA3 marker gene was used for silencing assays to determine URA3 expression in complete SC medium (Formedium ${ }^{\mathrm{TM}}$ ) or $\mathrm{SC}$ URA Drop-Out media (Formedium ${ }^{\mathrm{TM}}$ ). HIS1, ARG4, and NAT marker genes were used to replace both copies of SIR2, SET1, and JHD2 genes. PCR was used for screening of positive transformants. Oligonucleotides and plasmids used for strain constructions are listed in Supplementary Tables S2 and S3, respectively.

\section{Silencing Assay}

Growth analyses were performed using a plate reader (SpectrostarNano, BMG labtech) in 96 well plate formats at $30^{\circ} \mathrm{C}$. For each silencing assay in 96 well plate format, 1:100 


\section{A}

$\mathrm{Chr}$

Orf19.599 CENcoreR

1 Orf19.4439 $_{\text {CENcorel }}^{\mathrm{IR} 1}$

epsilon LTR

2 OENcore2 $_{\text {Orf19.1601 }}$

zeta LTR

$3 \overbrace{\text { Orf19.6923 }}$ CENcore3 $\underbrace{}_{\text {Orf19.2812 }}$

4 Orf19.3821 $_{\text {CENcore4 }}^{\text {IR4 Orf19.3820 }}$

5 Orf19.3161

Orf19.3160 IR6 IR6
IR6

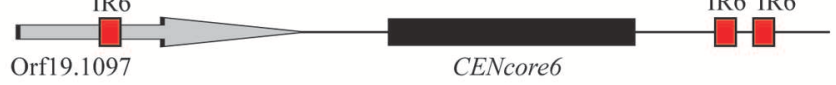

$7<_{\text {Orfl } 19.6524}$

B

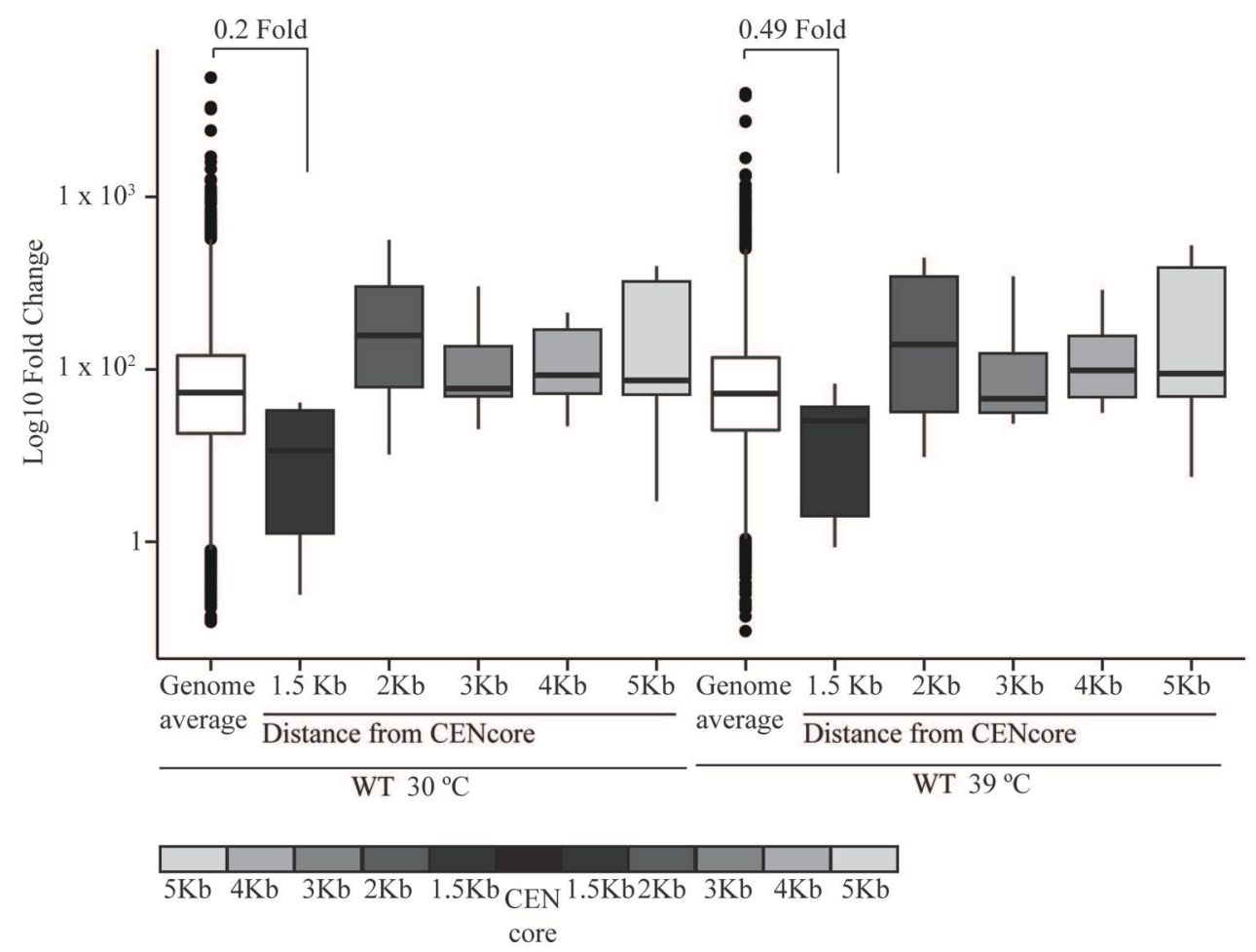

FIGURE 1 | Continued 


\section{FIGURE 1 | Continued}

Genes flanking centromeres display reduced expression. (A) Schematic of Candida albicans centromeres. On each chromosome, black squares indicate centromere core regions (CENcore). Red squares indicate inverted repeats (IRs). Orange empty squares indicate Long Terminal Repeats (LTRs). Empty arrows indicate tRNA codons. Gray arrows indicate ORF and their transcription orientation. (B) RNA deep-sequencing of WT isolates at $30^{\circ} \mathrm{C}$ and $39^{\circ} \mathrm{C}$. Boxplots represent normalized read counts (FPKM) of median genome expression compare to the median of all CENcore proximal genes from 1.5 to 5 Kb flanking both sides of CENcore regions on all chromosomes.

dilution of and overnight culture was inoculated in a final volume of $95 \mu \mathrm{l}$ of SC or SC-URA media to reach a concentration of 60 cells/ $/$ l. Growth was assessed by measuring $A_{600}$, using the following conditions: $\mathrm{OD}_{600 \mathrm{~nm}}, 616$ cycle time, three flashes per well, $700 \mathrm{rpm}$ shaking frequency, orbital shaking mode, $545 \mathrm{~s}$ additional shaking time after each cycle $0.5 \mathrm{~s}$ post delay, for $44 \mathrm{~h}$. Graphs represent data from three biological replicates. Error bars: standard deviation (SD) of three biological replicates generated from three independent cultures of the same strain. Data was processed using SpectrostarNano MARS software and Microsoft Excel.

\section{RNA Extraction and cDNA Synthesis}

RNA was extracted from $\log 2$ exponential cultures $\left(\mathrm{OD}_{600 \mathrm{~nm}}=1.4\right)$ using a yeast RNA extraction kit (E.Z.N.A. ${ }^{\circledR}$ Isolation Kit RNA Yeast, Omega Bio-Tek) following the manufacturer's instructions. RNA quality was checked by electrophoresis under denaturing conditions in $1 \%$ agarose, $1 \mathrm{X}$ HEPES, 6\% Formaldehyde (Sigma). RNA concentration was measured using a NanoDrop ND-1000 Spectrophotometer. cDNA synthesis was performed using iScript ${ }^{\mathrm{TM}}$ Reverse Transcription Supermix for RT-qPCR (Bio-Rad) following manufacturer's instructions and a Bio-Rad CFXConnect ${ }^{\mathrm{TM}}$ Real-Time System.

\section{High-throughput RNA Sequencing}

Strand-specific cDNA Illumina Barcoded Libraries were generated from $1 \mu \mathrm{g}$ of total RNA extracted from wt and $\operatorname{sir} 2 \Delta / \Delta$ strains and sequenced with an Illumina iSeq2000 platform. Illumina Library and Deep-sequencing was performed by the Genomics Core Facility at EMBL (Heidelberg, Germany). Raw reads were analyzed using TopHat algorithm following the RNA deep sequencing analysis pipeline described (Trapnell et al., 2013) using Galaxy ${ }^{1}$ and Linux platform. Heatmaps and boxplot graphs were generated with $\mathrm{R}^{2}$. RNA sequencing data are deposited into ArrayExpress (accession number E-MTAB-4622).

\section{Quantitative Chromatin ImmunoPrecipitation (qChIP)}

Quantitative Chromatin ImmunoPrecipitation (qChIP) was performed as described (Pidoux et al., 2004) with the following modifications: $5 \mathrm{ml}$ of an overnight culture grown in YPAD with extra uridine $(0.08 \mathrm{mg} / \mathrm{ml})$, diluted into fresh YPAD with extra uridine $(0.08 \mathrm{mg} / \mathrm{ml})$ and grown until OD600 nm of 1.4. Cells $(50 \mathrm{ml} / \mathrm{sample})$ were fixed with $1 \%$ Paraformaldehyde

${ }^{1}$ https://usegalaxy.org/

${ }^{2}$ http://www.r-project.org/
(Sigma) for $15 \mathrm{~min}$ at room temperature. Cells were lysed using acid-washed glass beads (Sigma) and a Disruptor genie ${ }^{\mathrm{TM}}$ (Scientific Industries) for $30 \mathrm{~min}$ at $4^{\circ} \mathrm{C}$. Chromatin was sheared to 500-1000 bp using a Bioruptor (Diagenode) for a total of $20 \mathrm{~min}\left(30 \mathrm{~s} \mathrm{ON}\right.$ and $\mathrm{OFF}$ cycle) at $4^{\circ} \mathrm{C}$. Immunoprecipitation was performed overnight at $4^{\circ} \mathrm{C}$ using $2 \mu \mathrm{L}$ of antibody antiH3K4me2 (Active Motif- Cat Number: 39141), anti-H3K9ac (Active Motif- Cat Number: 39137), and anti-H4K16ac (Active Motif- Cat Number: 39167) and $25 \mu$ l of Protein G magnetic beads (Dynal - InVitrogen). DNA was eluted with a $10 \%$ slurry of Chelex 100-resin (Bio-Rad) using the manufacturer's instructions.

\section{qPCR Reactions}

Primers used are listed in Supplementary Table S2. Realtime qPCR and RT-qPCR were performed in the presence of SYBR Green (Bio-Rad) on a Bio-Rad CFXConnect ${ }^{\mathrm{TM}}$ Real-Time System. Data was analyzed with Bio-Rad CFX Manager 3.1 software and Microsoft Excel. Enrichments were calculated as the percentage ratio of specific IP over input for qChIP analysis and as enrichment over actin for RT-qPCR. Histograms represent data from three biological replicates. Error bars: SD of three biological replicates generated from three independent cultures of the same strain.

\section{RESULTS}

\section{Expression Level of Centromere-Proximal Genes Is Low}

Heterochromatin represses expression of associated and proximal genes (Freitas-Junior et al., 2005; Kaur et al., 2005; Hansen et al., 2006; Merrick and Duraisingh, 2006). Therefore, if the pericentromeric regions of C. albicans were assembled into heterochromatin, genes in proximity to these regions would be poorly expressed. To test this hypothesis, we isolated RNA from wild-type (WT) cells grown at a temperature relevant for growth of C. albicans on the skin $\left(30^{\circ} \mathrm{C}\right)$ and at a temperature mimicking fever in the host $\left(39^{\circ} \mathrm{C}\right)$ and performed RNA-seq analyses. FPKM (fragments per kilobase of exons per million mapped reads) values were determined for each annotated gene. We then calculated the FPKM for genes in cumulative bins of $1 \mathrm{~kb}$ from the centromeres and compared these values to the genome-wide average (Figure 1B). This analysis reveals that genes in proximity to centromeres $(0$ to $1.5 \mathrm{~kb})$ are less expressed compared with the genome-wide average $\left(p\right.$-value $\left.=2.2 \times 10^{-16}\right)$. These data suggest that pericentromeric and centromeric regions impose a weak transcriptionally repressive environment. 


\section{A Marker Gene Inserted at Pericentromeric Repeats Is Partially Repressed}

Heterochromatin assembled onto repetitive DNA represses the transcription of marker genes inserted in their proximity (Henikoff and Dreesen, 1989; Gottschling et al., 1990; Bryk et al., 1997; Smith and Boeke, 1997). We have previously shown that, also in C. albicans, heterochromatin silences inserted marker genes (Freire-Benéitez et al., 2016). Our RNA-seq analyses suggest that regions proximal to a centromere impose a weak transcriptional repressive environment (Figure 1B). To test whether C. albicans pericentromeres impose transcriptional silencing dependently or independently of the presence of DNA repeats, we integrated the URA3 marker gene into the pericentromeric regions of centromeres surrounded by DNA repeats (peri-CEN4:URA3 ${ }^{+}$and peri-CEN5:URA3 ${ }^{+}$) and into the pericentromeric regions of CEN7, lacking DNA repeats (periCEN7:URA3 ${ }^{+}$). (Figure 2A). To investigate whether the URA3 marker gene is transcriptionally silenced when inserted at these locations, strains were grown in non-selective (N/S) medium and in medium lacking uridine (-Uri) in which only cells expressing sufficient Ura3 protein are able to grow. Silencing of URA3 is expected to result in slower growth in -Uri medium compared to N/S. However, none of the strains grew poorly in -Uri medium compared to N/S medium (Figure $2 B$ ). In contrast, quantitative reverse transcriptase analysis $(q R T-P C R)$ reveals that the levels of URA3 mRNA for the peri-CEN4:URA3 ${ }^{+}$, peri-CEN5:URA3 ${ }^{+}$, and peri-CEN7:URA3 ${ }^{+}$strains were significantly lower than the $U R A 3$ euchromatic gene (Figure 2C). Therefore, pericentromeric regions impose a weak transcriptional silencing that can only be detected at the RNA level independently of the presence of repetitive elements.

\section{Pericentromeric Repeats Are Assembled Into an Intermediate Chromatin State Bearing Features of Both Euchromatin and Heterochromatin}

We have shown that, in C. albicans, heterochromatin regions are assembled into nucleosomes that are hypoacetylated on $\mathrm{H} 3 \mathrm{~K} 9$ and H4K16 and hypomethylated on H3K4 (Freire-Benéitez et al., 2016). To assess whether pericentromeric regions are associated with heterochromatic histone marks, we monitored by $q \mathrm{ChIP}$ the presence of $\mathrm{H} 3 \mathrm{~K} 9 \mathrm{Ac}, \mathrm{H} 4 \mathrm{~K} 16 \mathrm{Ac}$, and $\mathrm{H} 3 \mathrm{~K} 4 \mathrm{me}$ at pericentromeric regions surrounding CEN4, CEN5, and CEN7 (Figure 3A). As a control, the chromatin state associated with the euchromatic ACT1 locus was analyzed. We find that all pericentromeric regions analyzed are assembled into chromatin that is highly acetylated on $\mathrm{H} 3 \mathrm{~K} 9$ and $\mathrm{H} 4 \mathrm{~K} 16$ as levels of these two histone modifications are similar to levels detected at the active and euchromatic locus ACT1 (Figures 3B,D, and F). High levels of H4K16 acetylation are also found at the central core region assembled into CENP-A chromatin (Figure 3). In contrast, pericentromeric chromatin is hypomethylated on $\mathrm{H} 3 \mathrm{~K} 4$, a chromatin state more similar to heterochromatic regions and different from the euchromatic ACT1 locus
(Figures 3B,D, and F). Thus, pericentromeric regions have only one of the three marks (H3K4 hypomethylation) associated with classic heterochromatin. Importantly, this chromatin state marks pericentromeric regions independently of the presence of repeats as pericentromeres with DNA repeats (CEN4 and CEN5) and without (CEN7) are associated with a similar histone modifications pattern. We concluded that C. albicans pericentromeres are not assembled into classical transcriptionally silent heterochromatin but they are associated with an intermediate chromatin state bearing features of euchromatin (high histone acetylation) and heterochromatin (H3K4 hypomethylation).

\section{The Chromatin State Associated with Pericentromeric Regions Is Independent of the Histone Deacetylase Sir2}

The HDAC Sir2 specifically deacetylates H3K9 and H4K16 and it is required for heterochromatin assembly across the eukaryotic kingdom (Rusche et al., 2003). We have shown that C. albicans Sir2 is necessary for heterochromatin integrity at the $r D N A$ locus and telomeric regions via deacetylation of $\mathrm{H} 3 \mathrm{~K} 9$ and H4K16 (Freire-Benéitez et al., 2016). To assess whether Sir2 contributes to the chromatin and transcriptional state of pericentromeric regions, we isolated RNA from WT and sir2 $\Delta / \Delta$ cells and performed RNA-seq analyses. FPKM values were determined for all genes proximal to CEN repeats and compared between sir2 $\Delta / \Delta$ and WT strains. Upon deletion of the SIR2 gene, we did not observe any clear effect on expression of CEN proximal genes (Figure 4A). Only 2 out of the 12 genes located in proximity $(<1.5 \mathrm{~Kb})$ of centromeres were expressed more than 2 fold in sir2 $\Delta / \Delta$ isolates compared to WT cells (Table 1). Therefore Sir2 does not contribute to the poor expression of CEN-proximal genes. In agreement with these results, deletion of the SIR2 gene does not increase the levels of $\mathrm{H} 3 \mathrm{~K} 9$ and H4K16 acetylation associated with pericentromeric region on chromosome five as revealed by $q$-ChIP analyses (Figure 4C).

\section{The Histone Methyltransferase Set1, But Not the Histone Demethylase Jhd2, Contributes to the Chromatin State Associated with Pericentromeres}

Coordination of activities between histone methyltransferases and histone demethylases ensures the right methylation level associated with euchromatic and heterochromatic loci. Therefore, specific histone methyltransferases and demethylases might be important for maintaining the H3K4 hypomethylated state associated with C. albicans pericentromeres. The C. albicans genome encodes for the H3K4 methyltransferase Set1 (Raman et al., 2006) and for the putative H3K4 demethylase Kmd5/Jhd2 (orf19.5651). In S. cerevisiae, both proteins have been implicated in transcriptional silencing and heterochromatin formation (Briggs et al., 2001; Ingvarsdottir et al., 2007; Ryu and Ahn, 2014). To assess whether Set1 and/or Jhd 2 contribute to the chromatin state associated with $C$. albicans pericentromeric regions, we deleted both copies of the SET1 and JHD2 genes 


\section{A}

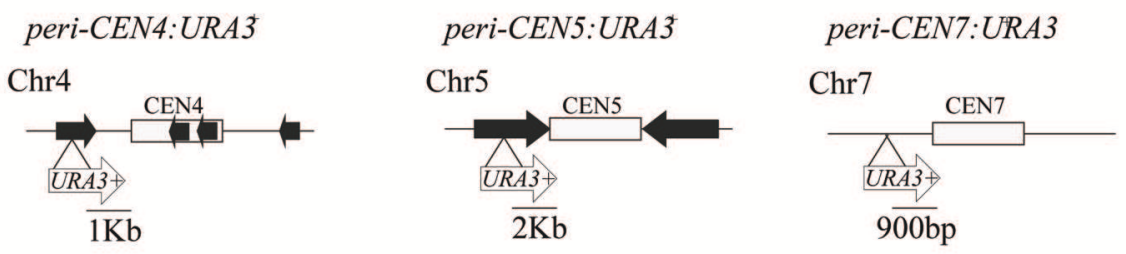

B

Silencing assay

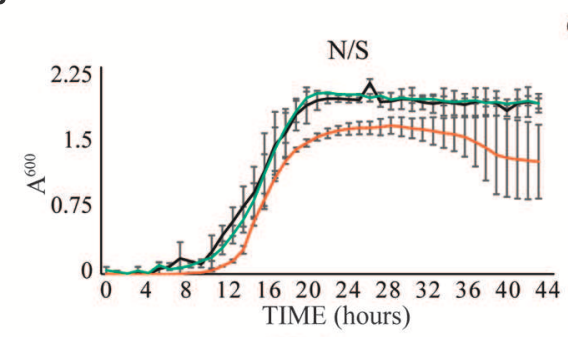

CEN4

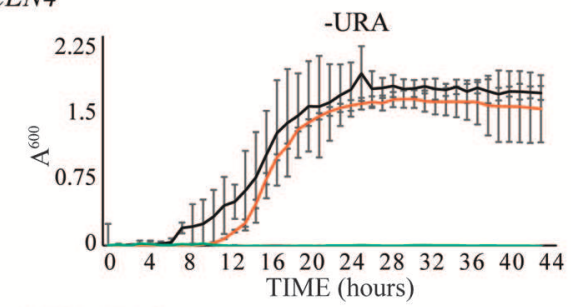

- $U R A 3^{+}$

- : peri-CEN4:URA3

— $\operatorname{ura} 3 \Delta$ ura3 $\Delta$

CEN5
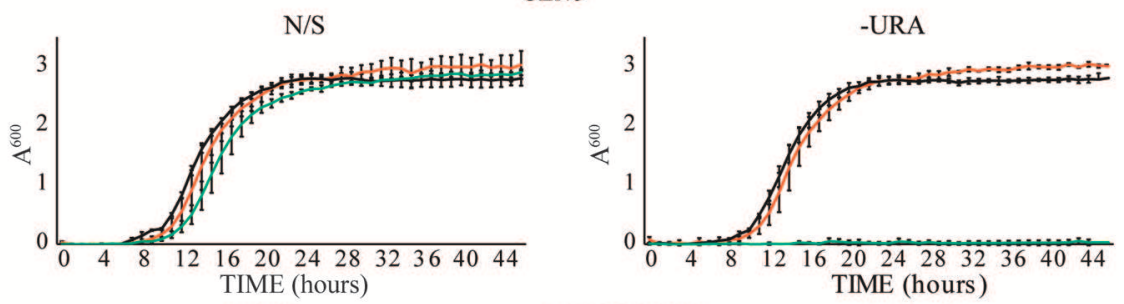

- $U R A 3^{+}$

— : peri-CEN5:URA3+

- $\operatorname{ura} 3 \Delta$ ura3 $\Delta$

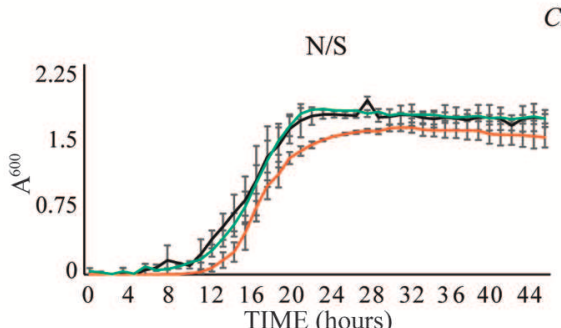

CEN7

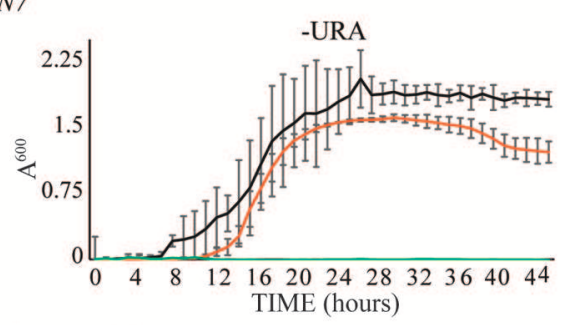

- U URA3

- : peri-CEN7:URA3

- $\operatorname{ura3} 3 \mathrm{Aura} 3 \Delta$

C

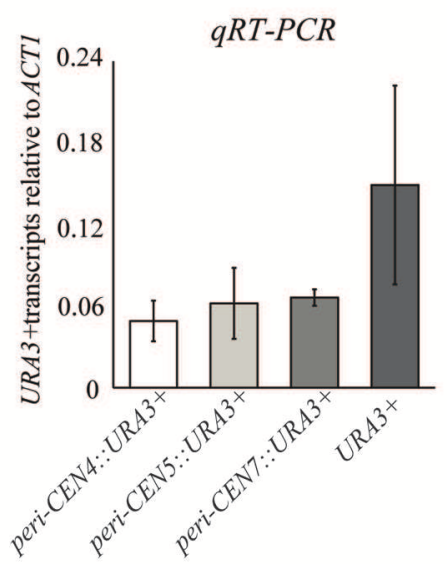




\section{FIGURE 2 | Continued}

Weak transcriptional silencing of a URA3 marker gene integrated at pericentromeric region. (A) Schematic of peri-CEN4:URA3 ${ }^{+}$, peri-CEN5:URA3 ${ }^{+}$, and peri-CEN7:URA3 ${ }^{+}$reporter strains. Distance from the URA3 marker gene to each CENcore is indicated in $\mathrm{Kb}$ (B) Silencing assay of the peri-CEN4:URA3 ${ }^{+}$,

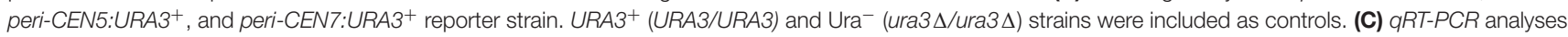
to measure URA3 ${ }^{+}$transcript levels of the peri-CEN4:URA3 ${ }^{+}$, peri-CEN5:URA3 ${ }^{+}$, and peri-CEN7:URA3 ${ }^{+}$reporter strain relative to actin transcript levels (ACT1). Error bars in each panel: standard deviation (SD) of three biological replicates.
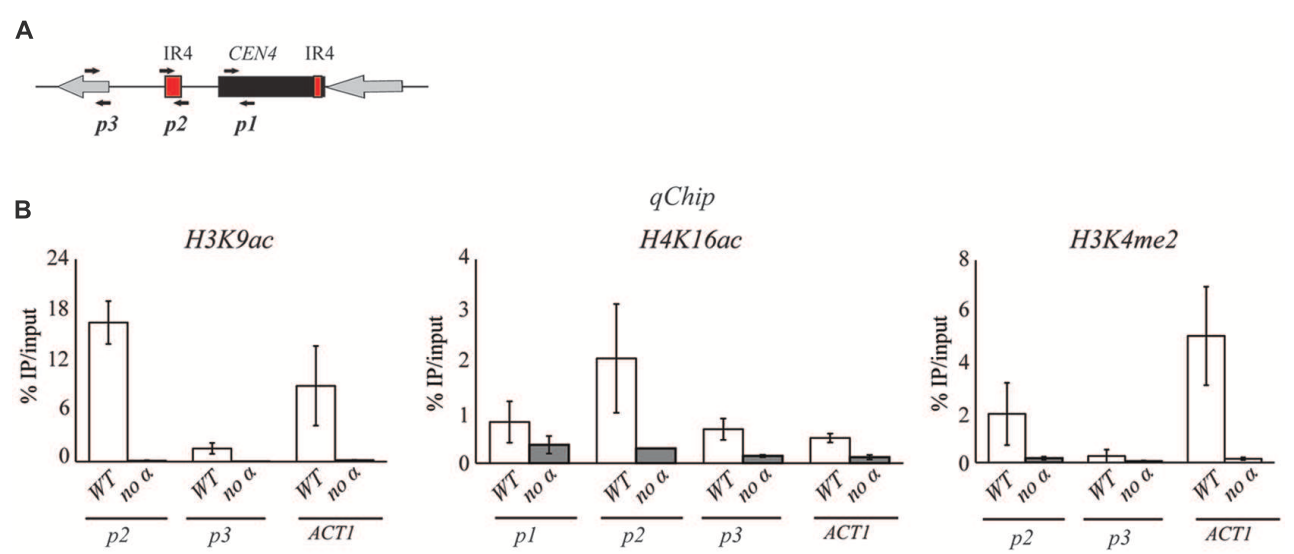

C

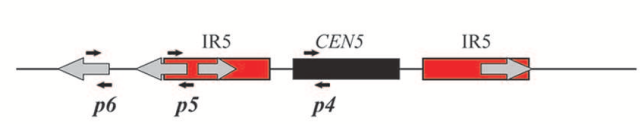

D
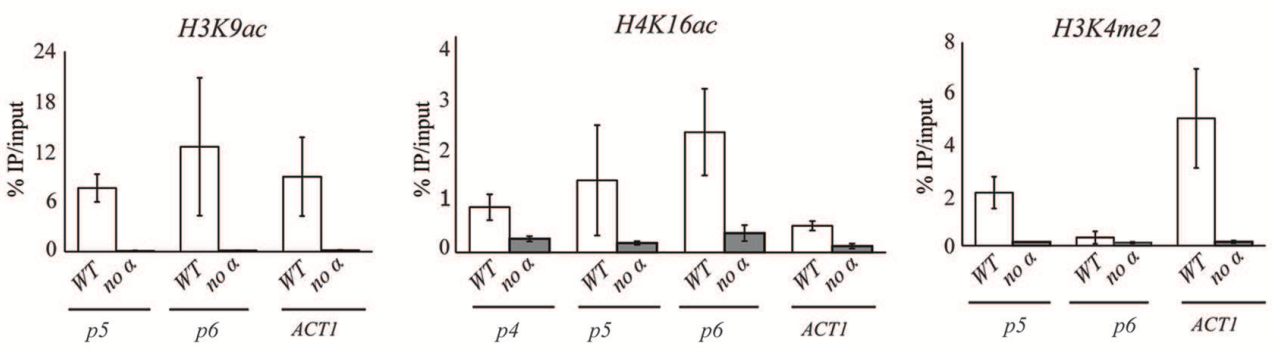

E

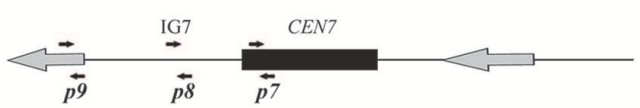

$\mathrm{F}$

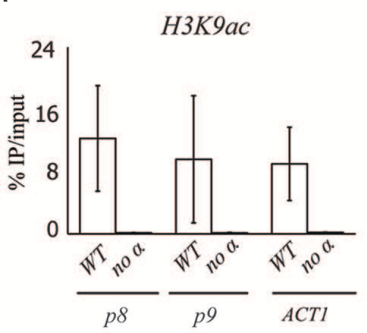

qChip

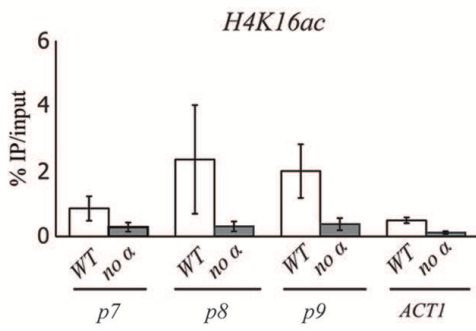

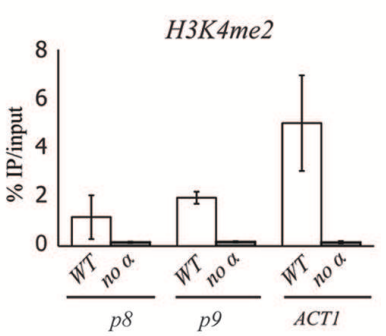

FIGURE 3 | The chromatin state of C. albicans centromeres. (A) Schematic of C. albicans CEN4. Black arrows indicate the primers used to study the chromatin pattern associated with CEN4 (B) qChIP to detect H3K9ac and H3K4me2 levels associated with CEN 4. ACT1 was used as a control. (C) Schematic of C. albicans CEN5. Black arrows indicate the primers used to study the chromatin pattern associated with CEN5. (D) qChIP to detect H3K9ac and H3K4me2 levels associated with CEN5. ACT1 was used as a control. (E) Schematic of C. albicans CEN7. Black arrows indicate the primers used to study the chromatin pattern associated with CEN7. (F) qChIP to detect H3K9ac, H4K16ac and H3K4me2 levels associated with CEN7. ACT1 was used as a control. Error bars in each panel: $\mathrm{SD}$ of three biological replicates. 


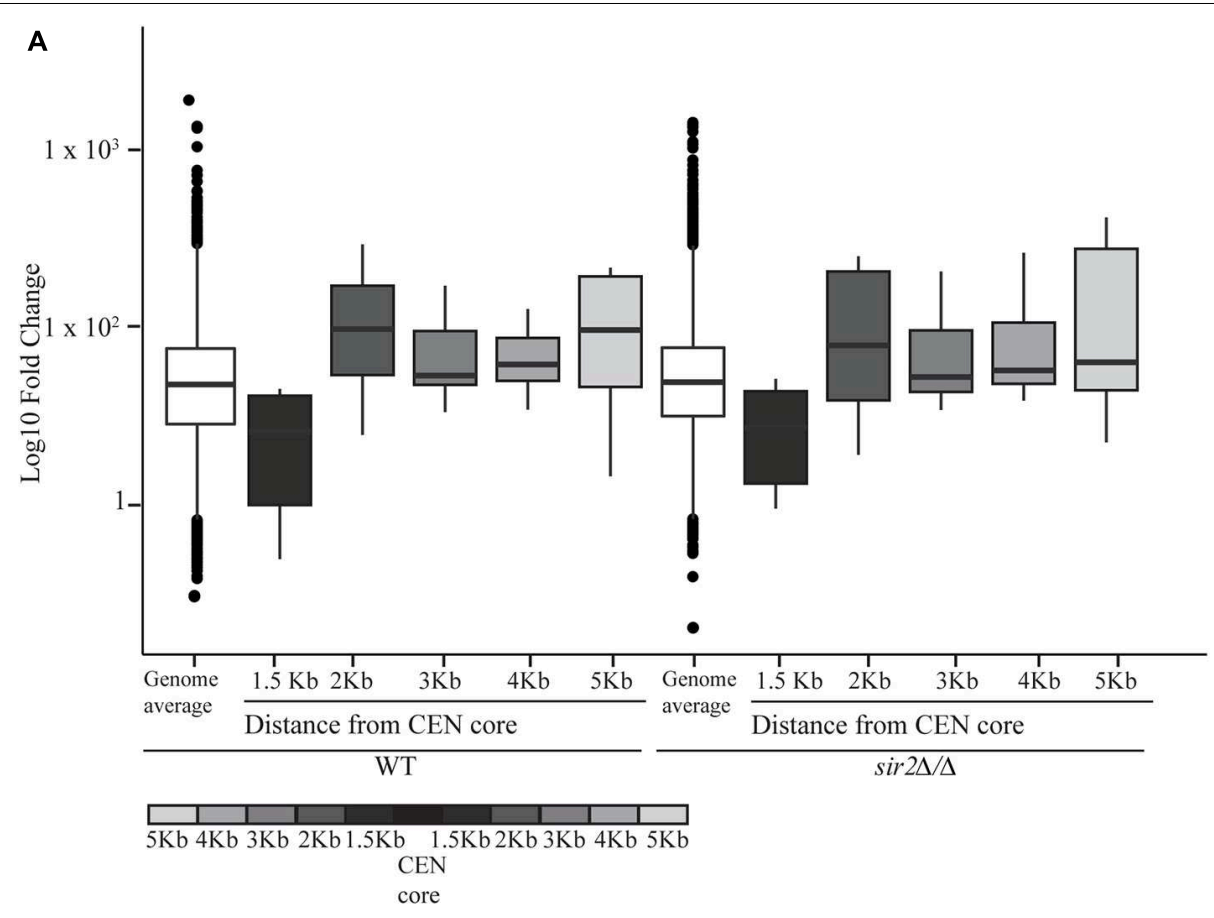

B

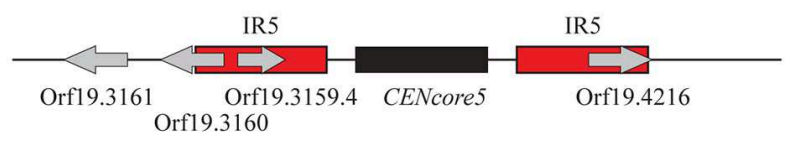

C

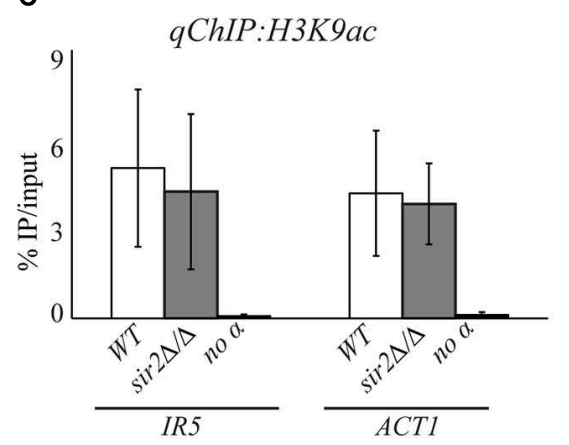

FIGURE 4 | The chromatin state associated with C. albicans centromeres is independent of the histone deacetylase (HDAC) Sir2. (A) RNA deep-sequencing of WT and sir2 $\Delta / \Delta$. Boxplots represent normalized read counts (FPKM) of median genome expression compare to the median of all $C E N$ proximal genes from 1.5 to $5 \mathrm{~Kb}$ (B) Schematic of C. albicans CEN5. Black arrows indicate the primers used to study the chromatin pattern associated with the IRs5 of CEN5. (C) qChIP to detect H3K9ac levels associated with the IRs5 of CEN5 and ACT1 in WT and sir2 $\Delta / \Delta$ strains. Error bars in each panel: SD of three biological replicates.

from WT cells and quantified $\mathrm{H} 3 \mathrm{~K} 4 \mathrm{me} 2$ levels by $q$ ChIP analyses. We find that Set1 is necessary for maintaining the low levels of $\mathrm{H} 3 \mathrm{~K} 4 \mathrm{me}$ associated with CEN5 repeats (Figure 5A) as $\mathrm{H} 3 \mathrm{~K} 4 \mathrm{me}$ dropped to background levels in set $1 \Delta / \Delta$ compared to WT cells (Figure $5 B$ ). In contrast, we find that Jhd2 is not required for maintaining the hypomethylated state associated with CEN5 (Figure 5A) repeats as $\mathrm{H} 3 \mathrm{~K} 4 \mathrm{me}$ levels did not change between $j h d 2 \Delta / \Delta$ and WT cells (Figure 5C).

\section{DISCUSSION}

This study is the first analysis of the chromatin state associated with pericentromeric regions in the human fungal pathogen C. albicans.

In many organisms, regional centromeres have a conserved modular structure despite the lack of a conserved DNA sequence. At these locations, CENP-A domains, sites of kinetochore assembly, are flanked or interspersed by DNA repeats assembled 
TABLE 1 | RNA deep-sequencing values of $1.5 \mathrm{~Kb}$ CEN proximal genes.

\begin{tabular}{|c|c|c|c|c|c|c|c|}
\hline ORF & Gene & CENcore & bp distance to CENcore & $W T$ FPKM & $\operatorname{sir} 2 \Delta / \Delta$ FPKM & (sir2 $\Delta / \Delta / W T$ ) FPKM Fold & Log2(Fold) \\
\hline orf19.1604 & - & 2 & 96 & 27.1566 & 36.3803 & 1.339649 & 0.421855 \\
\hline orf19.3820 & - & 4 & 51 & 0.913477 & 0.96385 & 1.055144 & 0.07744 \\
\hline orf19.3159.4 & - & 5 & 926 & 0.516172 & 2.34077 & 4.534864 & 2.18106 \\
\hline orf19.1097 & ALS2 & 6 & 1498 & 9.68354 & 39.5448 & 4.083713 & 2.029882 \\
\hline orf19.2124 & - & 6 & 765 & 20.2353 & 29.1543 & 1.440764 & 0.526838 \\
\hline orf19.599 & - & $\mathrm{R}$ & 102 & 1.24606 & 1.76585 & 1.417147 & 0.502991 \\
\hline orf19.598 & - & $\mathrm{R}$ & 1126 & 0.209874 & 1.269 & 6.046485 & -1.16192 \\
\hline
\end{tabular}

ORFs include all genes within $1.5 \mathrm{~Kb}$ of a centromere core. Values are indicated in FPKM for WT and sir2 $\Delta / \Delta$ strains, including FPKM fold increase and Log2 fold increase of sir2 $\Delta / \Delta$ strain over WT strain.

A

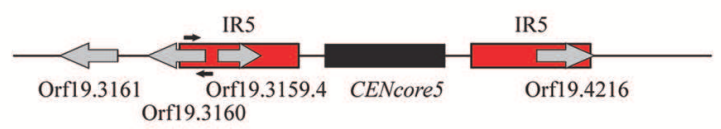

B

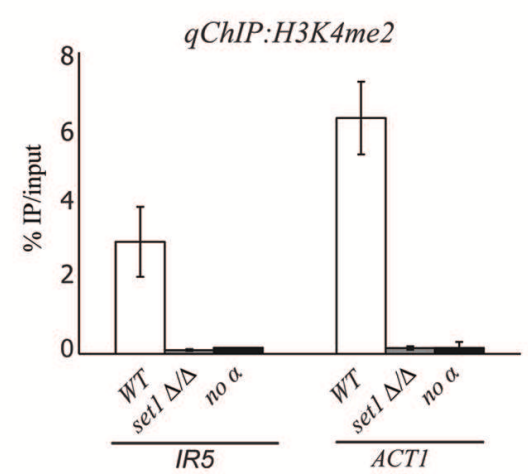

C

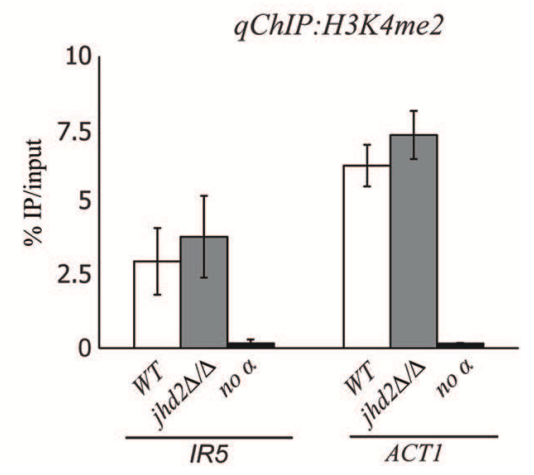

FIGURE 5 | The chromatin state associated with pericentromeric regions is independent of the histone methyltransferase Set1 and the histone demethylase Jhd2. (A) Schematic of C. albicans CEN5. Black arrows indicate the primers used to study the chromatin pattern associated with the IRs5 of CEN5. (B) qChIP to detect H3K4me2 levels associated with the IRs5 of CEN5 and ACT1 in WT and set1 $\Delta / \Delta$ strains. (C) $q C h I P$ to detect H3K4me2 levels associated with the IRs5 of CEN5 and ACT1 in WT and jhd2 $\Delta / \Delta$ strains. Error bars in each panel: SD of three biological replicates.

into heterochromatin (Buscaino et al., 2010). Both CENP-A chromatin and pericentromeric heterochromatin are inhibitory to transcription as illustrated by silencing of inserted marker genes (Allshire et al., 1994; Ketel et al., 2009).

The small C. albicans regional centromeres are composed of a CENP-A central core surrounded by pericentromeric regions with different sequence and organization. CENP-A chromatin represses transcription of embedded marker genes (Ketel et al., 2009). Here, we show that, despite the absence of canonical heterochromatin, C. albicans pericentromeric regions weakly repress transcription. These conclusions are supported by two observations. Firstly, RNA-sequence analysis highlights that genes located in proximity $(<1.5 \mathrm{~Kb})$ to centromeres are less expressed than the genome-wide average (Figure 1B). Secondly, a URA3 marker gene inserted into the pericentromeric region of chromosomes 4, 5, and 7 is weakly silenced. This weak silencing is observed when URA3 RNA levels are measured by $q R T-P C R$ but no silencing is detected by growing strains in -URA medium (Figure 2). This suggests that the reduced expression of the $U_{R A 3}{ }^{+}$marker gene is sufficient to confer a URA ${ }^{+}$growth phenotype.

Candida albicans lacks the heterochromatic structure that is normally associated with regional centromeres because the $C$. albicans genome does not encode for SuVar 3-9, the H3K9-specific methyltransferase, or for HP-1, the chromodomain protein responsible for assembly and spreading of centromeric heterochromatin. We have recently shown that transcriptionally silenced heterochromatin exists in C. albicans and it is associated with the rDNA locus and telomeric regions (Freire-Benéitez et al., 2016). This heterochromatic state, 
similarly to $S$. cerevisiae, is characterized by hypoacetylated nucleosomes that are hypomethylated on H3K4 (FreireBenéitez et al., 2016). Here, we show that $C$. albicans pericentromeric regions are not associated with heterochromatin but with an intermediate chromatin state bearing features of both euchromatin and heterochromatin. Nucleosomes at pericentromeric regions are highly acetylated, as observed in euchromatin, and hypomethylated on $\mathrm{H} 3 \mathrm{~K} 4$, as observed in heterochromatin (Figure 3). Consistently, we find that deletion of the HDAC Sir2 does not perturb the chromatin state of pericentromeric regions (Figure 4).

Given the lack of canonical heterochromatin at pericentromeric regions, it is still unclear what drives the weak transcriptional silencing associated with these regions. We envisage two possible scenarios. It is possible that low levels of CENP-A, undetectable by ChIP analyses, associate with pericentromeric regions driving transcriptional silencing. In support of this hypothesis, it is well known that CENPA chromatin is inhibitory to transcription. In addition, it has been observed that, following deletion of endogenous centromeric sequences, neocentromeres often form immediately adjacent to the site of the excised native centromeres (Ketel et al., 2009; Shang et al., 2013; Thakur and Sanyal, 2013). These data suggest that low levels of CENP-A might be present at pericentromeric regions and might be sufficient to nucleate new centromeres. In agreement with this hypothesis, it has been shown that a pool of free CENP-A accessory molecules is present in vicinity of centromeres. These accessory molecules do not nucleate kinetochore assembly but could allow for rapid incorporation of CENP-A in the event of eviction at the centromere (Haase et al., 2013). Therefore, it is possible that CENP-A accessory molecules drive the transcriptional silencing associated with pericentromeric repeats. Finally, it has been shown that, following neocentromere formation, assembly of CENP-A into transcribed regions is sufficient to repress gene expression (Shang et al., 2013).

\section{REFERENCES}

Allshire, R. C., Javerzat, J. P., Redhead, N. J., and Cranston, G. (1994). Position effect variegation at fission yeast centromeres. Cell 76, 157-169. doi: 10.1016/0092-8674(94)90180-5

Baum, M., Ngan, V. K., and Clarke, L. (1994). The centromeric K-type repeat and the central core are together sufficient to establish a functional Schizosaccharomyces pombe centromere. Mol. Biol. Cell 5, 747-761. doi: 10.1091/mbc.5.7.747

Baum, M., Sanyal, K., Mishra, P. K., Thaler, N., and Carbon, J. (2006). Formation of functional centromeric chromatin is specified epigenetically in Candida albicans. Proc. Natl. Acad. Sci. U.S.A. 103, 14877-14882. doi: 10.1073/pnas.0606958103

Bernard, P., Maure, J. F., Partridge, J. F., Genier, S., Javerzat, J. P., and Allshire, R. C. (2001). Requirement of heterochromatin for cohesion at centromeres. Science 294, 2539-2542. doi: 10.1126/science.1064027

Briggs, S. D., Bryk, M., Strahl, B. D., Cheung, W. L., Davie, J. K., Dent, S. Y., et al. (2001). Histone H3 lysine 4 methylation is mediated by Set1 and required for cell growth and rDNA silencing in Saccharomyces cerevisiae. Genes Dev. 15, 3286-3295. doi: 10.1101/gad.940201

Bryk, M., Banerjee, M., Murphy, M., Knudsen, K. E., Garfinkel, D. J., and Curcio, M. J. (1997). Transcriptional silencing of Tyl elements in
An alternative hypothesis is that the chromatin state associated with pericentromeric regions is sufficient to drive transcriptional silencing. We find the pericentromeric regions are hypomethylated on $\mathrm{H} 3 \mathrm{~K} 4$, an epigenetic heterochromatic mark. It is possible that this mark is sufficient to drive transcriptional repression even in the presence of acetylated histones. In support of this hypothesis, it is well established that methylation of $\mathrm{H} 3 \mathrm{~K} 4$ correlates with gene expression and heterochromatic regions are marked by H3K4 hypomethylation (Fischle et al., 2003; Lachner et al., 2004).

The biology of $C$. albicans DNA repeats, including pericentromeric regions, is still poorly understood. Future studies will reveal whether and how the novel chromatin state associated with these pericentromeric regions controls centromere function and/or identity.

\section{FUNDING}

This work was supported by BBSRC (BB/L008041/1 to AB), MRC (MR/M019713/1 to RJP and AB), and a Royal Society Research Grant (RG130149 to $\mathrm{AB}$ ).

\section{ACKNOWLEDGMENTS}

We thank J. Berman and A. Mitchell for strains and materials and S. Gourlay for critical reading of the manuscript. We thank the Gene Core Facility at EMBL (Heidelberg-Germany) for RNA Sequencing and M. Wass for support with the RNA Seq analyses.

\section{SUPPLEMENTARY MATERIAL}

The Supplementary Material for this article can be found online at: http://journal.frontiersin.org/article/10.3389/fmicb. 2016.00759

the RDN1 locus of yeast. Genes Dev. 11, 255-269. doi: 10.1101/gad.11. 2.255

Bühler, M., and Gasser, S. M. (2009). Silent chromatin at the middle and ends: lessons from yeasts. EMBO J. 28, 2149-2161. doi: 10.1038/emboj. 2009.185

Buscaino, A., Allshire, R., and Pidoux, A. (2010). Building centromeres: home sweet home or a nomadic existence? Curr. Opin. Genet. Dev. 20, 118-126. doi: 10.1016/j.gde.2010.01.006

Chatterjee, G., Sankaranarayanan, S. R., Guin, K., Thattikota, Y., Padmanabhan, S., Siddharthan, R., et al. (2016). Repeat-associated fission yeast-like regional centromeres in the ascomycetous budding yeast Candida tropicalis. PLoS Genet. 12:e1005839. doi: 10.1371/journal.pgen.1005839

De Backer, M. D., Maes, D., Vandoninck, S., Logghe, M., Contreras, R., and Luyten, W. H. (1999). Transformation of Candida albicans by electroporation. Yeast 15, 1609-1618. doi: 10.1002/(SICI)1097-0061(199911)15:15<1609::AIDYEA485>3.3.CO;2-P

Fischle, W., Wang, Y., and Allis, C. D. (2003). Histone and chromatin cross-talk. Curr. Opin. Cell Biol. 15, 172-183. doi: 10.1016/S0955-0674(03) 00013-9

Folco, H. D., Pidoux, A. L., Urano, T., and Allshire, R. C. (2008). Heterochromatin and RNAi are required to establish CENP-A chromatin at centromeres. Science 319, 94-97. doi: 10.1126/science.1150944 
Freire-Benéitez, V., Price, R. J., Tarrant, D., Berman, J., and Buscaino, A. (2016). Candida albicans repetitive elements display epigenetic diversity and plasticity. Sci. Rep. 6, 22989. doi: 10.1038/srep22989

Freitas-Junior, L. H., Hernandez-Rivas, R., Ralph, S. A., Montiel-Condado, D., Ruvalcaba-Salazar, O. K., Rojas-Meza, A. P., et al. (2005). Telomeric heterochromatin propagation and histone acetylation control mutually exclusive expression of antigenic variation genes in malaria parasites. Cell 121, 25-36. doi: 10.1016/j.cell.2005.01.037

Gottschling, D. E., Aparicio, O. M., Billington, B. L., and Zakian, V. A. (1990). Position effect at $S$. cerevisiae telomeres: reversible repression of Pol II transcription. Cell 63, 751-762. doi: 10.1016/0092-8674(90)90141-Z

Haase, J., Mishra, P. K., Stephens, A., Haggerty, R., Quammen, C., Taylor, R. M., et al. (2013). A 3D map of the yeast kinetochore reveals the presence of core and accessory centromere-specific histone. Curr. Biol. 23, 1939-1944. doi: 10.1016/j.cub.2013.07.083

Hansen, K. R., Ibarra, P. T., and Thon, G. (2006). Evolutionary-conserved telomere-linked helicase genes of fission yeast are repressed by silencing factors, RNAi components and the telomere-binding protein Tazl. Nucleic Acids Res. 34, 78-88. doi: 10.1093/nar/gkj415

Henikoff, S., and Dreesen, T. D. (1989). Trans-inactivation of the Drosophila brown gene: evidence for transcriptional repression and somatic pairing dependence. Proc. Natl. Acad. Sci. U.S.A. 86, 6704-6708. doi: 10.1073/pnas.86.17.6704

Ingvarsdottir, K., Edwards, C., Lee, M. G., Lee, J. S., Schultz, D. C., Shilatifard, A., et al. (2007). Histone H3 K4 demethylation during activation and attenuation of GAL1 transcription in Saccharomyces cerevisiae. Mol. Cell. Biol. 27, 7856-7864. doi: 10.1128/MCB.00801-07

Kapoor, S., Zhu, L., Froyd, C., Liu, T., and Rusche, L. N. (2015). Regional centromeres in the yeast Candida lusitaniae lack pericentromeric heterochromatin. Proc. Natl. Acad. Sci. U.S.A. 112, 12139-12144. doi: 10.1073/pnas.1508749112

Kaur, R., Domergue, R., Zupancic, M. L., and Cormack, B. P. (2005). A yeast by any other name: Candida glabrata and its interaction with the host. Curr. Opin. Microbiol. 8, 378-384. doi: 10.1016/j.mib.2005.06.012

Ketel, C., Wang, H. S. W., McClellan, M., Bouchonville, K., Selmecki, A., Lahav, T., et al. (2009). Neocentromeres form efficiently at multiple possible loci in Candida albicans. PLoS Genet. 5:e1000400. doi: 10.1371/journal.pgen.1000400

Kouzarides, T. (2007). Chromatin modifications and their function. Cell 128, 693-705. doi: 10.1016/j.cell.2007.02.005

Kueng, S., Oppikofer, M., and Gasser, S. M. (2013). SIR proteins and the assembly of silent chromatin in budding yeast. Annu. Rev. Genet. 47, 275-306. doi: 10.1146/annurev-genet-021313-173730

Lachner, M., Sengupta, R., Schotta, G., and Jenuwein, T. (2004). Trilogies of histone lysine methylation as epigenetic landmarks of the eukaryotic genome. Cold Spring Harb. Symp. Quant. Biol. 69, 209-218. doi: 10.1101/sqb.2004.69.209

Merrick, C. J., and Duraisingh, M. T. (2006). Heterochromatin-mediated control of virulence gene expression. Mol. Microbiol. 62, 612-620. doi: 10.1111/j.13652958.2006.05397.x

Mishra, P. K., Baum, M., and Carbon, J. (2007). Centromere size and position in Candida albicans are evolutionarily conserved independent of DNA sequence heterogeneity. Mol. Genet. Genomics 278, 455-465. doi: 10.1007/s00438-0070263-8

Nonaka, N., Kitajima, T., Yokobayashi, S., Xiao, G., Yamamoto, M., Grewal, S. I. S., et al. (2002). Recruitment of cohesin to heterochromatic regions by Swi6/HP1 in fission yeast. Nat. Cell Biol. 4, 89-93. doi: 10.1038/ncb739

Padmanabhan, S., Thakur, J., Siddharthan, R., and Sanyal, K. (2008). Rapid evolution of Cse4p-rich centromeric DNA sequences in closely related pathogenic yeasts, Candida albicans and Candida dubliniensis. Proc. Natl. Acad. Sci. U.S.A. 105, 19797-19802. doi: 10.1073/pnas.0809770105

Pidoux, A., Mellone, B., and Allshire, R. (2004). Analysis of chromatin in fission yeast. Methods 33, 252-259. doi: 10.1016/j.ymeth.2003.11.021

Raman, S. B., Nguyen, M. H., Zhang, Z., Cheng, S., Jia, H. Y., Weisner, N., et al. (2006). Candida albicans SET1 encodes a histone 3 lysine 4 methyltransferase that contributes to the pathogenesis of invasive candidiasis. Mol. Microbiol. 60, 697-709. doi: 10.1111/j.1365-2958.2006.05121.x

Rusche, L. N., Kirchmaier, A. L., and Rine, J. (2003). The establishment, inheritance, and function of silenced chromatin in Saccharomyces cerevisiae. Annu. Rev. Biochem. 72, 481-516. doi: 10.1146/annurev.biochem.72.121801.161547

Ryu, H.-Y., and Ahn, S. (2014). Yeast histone H3 lysine 4 demethylase Jhd2 regulates mitotic ribosomal DNA condensation. BMC Biol. 12:75. doi: 10.1186/s12915-014-0075-3

Sanyal, K., Baum, M., and Carbon, J. (2004). Centromeric DNA sequences in the pathogenic yeast Candida albicans are all different and unique. Proc. Natl. Acad. Sci. U.S.A. 101, 11374-11379. doi: 10.1073/pnas.0404318101

Shang, W. H., Hori, T., Martins, N. M. C., Toyoda, A., Misu, S., Monma, N., et al. (2013). Chromosome engineering allows the efficient isolation of vertebrate neocentromeres. Dev. Cell 24, 635-648. doi: 10.1016/j.devcel.2013.02.009

Shankaranarayana, G. D., Motamedi, M. R., Moazed, D., and Grewal, S. I. S. (2003). Sir2 regulates histone $\mathrm{H} 3$ lysine 9 methylation and heterochromatin assembly in fission yeast. Curr. Biol. 13, 1240-1246. doi: 10.1016/S0960-9822(03)00489-5

Smith, J. S., and Boeke, J. D. (1997). An unusual form of transcriptional silencing in yeast ribosomal DNA. Genes Dev. 11, 241-254. doi: 10.1101/gad.11.2.241

Strahl, B. D., and Allis, C. D. (2000). The language of covalent histone modifications. Nature 403, 41-45. doi: 10.1038/47412

Thakur, J., and Sanyal, K. (2013). Efficient neocentromere formation is suppressed by gene conversion to maintain centromere function at native physical chromosomal loci in Candida albicans. Genome Res. 23, 638-652. doi: 10.1101/gr.141614.112

Trapnell, C., Hendrickson, D. G., Sauvageau, M., Goff, L., Rinn, J. L., and Pachter, L. (2013). Differential analysis of gene regulation at transcript resolution with RNA-seq. Nat. Biotechnol. 31, 46-53. doi: 10.1038/nbt.2450

Westermann, S., Drubin, D. G., and Barnes, G. (2007). Structures and functions of yeast kinetochore complexes. Annu. Rev. Biochem. 76, 563-591. doi: 10.1146/annurev.biochem.76.052705.160607

Wilson, R. B., Davis, D., and Mitchell, A. P. (1999). Rapid hypothesis testing with Candida albicans through gene disruption with short homology regions. J. Bacteriol. 181, 1868-1874.

Wirén, M., Silverstein, R. A., Sinha, I., Walfridsson, J., Lee, H.-M., Laurenson, P., et al. (2005). Genomewide analysis of nucleosome density histone acetylation and HDAC function in fission yeast. EMBO J. 24, 2906-2918. doi: 10.1038/sj.emboj.7600758

Conflict of Interest Statement: The authors declare that the research was conducted in the absence of any commercial or financial relationships that could be construed as a potential conflict of interest.

Copyright $\odot 2016$ Freire-Benéitez, Price and Buscaino. This is an open-access article distributed under the terms of the Creative Commons Attribution License (CC BY). The use, distribution or reproduction in other forums is permitted, provided the original author(s) or licensor are credited and that the original publication in this journal is cited, in accordance with accepted academic practice. No use, distribution or reproduction is permitted which does not comply with these terms. 